OPEN ACCESS

Edited by:

Chunbo Chen,

Maoming People's Hospital, China

Reviewed by:

Miodrag Milenovic,

University of Belgrade, Serbia

Maria Chiara Maccarone

University Hospital of Padua, Italy

Christian Hofer

Otto Bock HealthCare Products

$\mathrm{GmbH}$, Austria

Masahide Hamaguchi,

Kyoto Prefectural University of

Medicine, Japan

*Correspondence:

Wellington Pereira Yamaguti wellington.psyamaguti@hsl.org.br

Specialty section:

This article was submitted to Intensive Care Medicine and

Anesthesiology,

a section of the journal

Frontiers in Medicine

Received: 17 August 2021 Accepted: 11 January 2022

Published: 16 February 2022

Citation:

Righetti RF, Grams ST, Costa WNdS,

Saraiva LT, Salles ICDd and Yamaguti WP (2022) Neuromuscular Electrical Stimulation in Patients With Severe COVID-19 Associated With

Sepsis and Septic Shock.

Front. Med. 9:751636.

doi: 10.3389/fmed.2022.751636

\section{Neuromuscular Electrical Stimulation in Patients With Severe COVID-19 Associated With Sepsis and Septic Shock}

\author{
Renato Fraga Righetti, Samantha Torres Grams, Wesla Neves da Silva Costa, \\ Leandro Teixeira Saraiva, Isabel Chateaubriand Diniz de Salles and \\ Wellington Pereira Yamaguti*
}

Hospital Sírio-Libanês, São Paulo, Brazil

Background: Neuromuscular electrical stimulation (NMES) can be applied to critically ill patients. However, its results on muscle strength and functionality in patients with COVID-19 are unknown.

Objective: Evaluate the effects of intervention with NMES on muscle mass and functionality of patients with severe COVID-19 associated with sepsis and septic shock.

Methods: Seven patients with COVID-19 associated with sepsis or septic shock were selected, but only 5 patients completed all days of the intervention with NMES. The intervention was performed by a single physiotherapist on 7 consecutive days in a daily session of $40 \mathrm{~min}$. The outcome measures were the femoris cross-sectional area; thickness of the anterior compartment of the quadriceps muscle; rectus femoris echogenicity; International Classification of Functioning, Disability, and Health (ICF)muscle strength; PFIT-s, DEMMI, and the SOMS; feasibility, and safety. The patients were evaluated on days 1,5 , and 8.

Results: The rectus femoris cross-sectional area decreased significantly from days 1 to 8 , but showed maintenance of the thickness of the anterior compartment of the quadriceps muscle from days 1 to 8 . The MRC score increased significantly from days 1 to 5 and kept this improvement until day 8 . All patients showed an increase in the MRC score and reduction of the ICF-muscle strength, meaning improved muscle strength from days 1 to 8 . The PFIT-s increased significantly from days 1 to 5 and improved until day 8 compared to day 5. DEMMI and SOMS score increased significantly on day 8 compared to days 1 and 5 .

Conclusion: Rehabilitation with NMES showed improvement in muscle strength and functionality of patients in this study with a potential protective effect on muscle mass loss in patients with critical COVID-19 associated with sepsis and septic shock. This study is the first report of the potential effects of neuromuscular electrical stimulation in patients with severe COVID-19 associated with sepsis and septic shock.

Keywords: COVID-19, sepsis, physiotherapy, neuromuscular electrical stimulation, muscle mass 


\section{INTRODUCTION}

The Coronavirus disease 2019 (COVID-19) is caused by novel Severe Acute Respiratory Syndrome Coronavirus 2 (SARS-CoV2) (1). The virus spread rapidly through the world population and several hospitals have produced guidelines for the respiratory management of these patients $(2,3)$. Most patients have a mild form of the disease, but $5 \%$ of patients present severe lung injury and required intensive care (4). These patients may develop ICU-acquired weakness (5).

Early mobilization in the intensive care unit (ICU) is proven to be effective in preventing muscle atrophy and functional disability. However, it is not necessarily applicable to all patients (6). Therefore, neuromuscular electrical stimulation (NMES) has been used as an additional rehabilitation strategy for critically ill patients (7). Studies using electrical muscle stimulation in septic patients have conflicting results depending on the titred stimulation frequency used. These studies showed that low stimulation frequency electrical stimulation was ineffective to preserve muscle mass (8) and high stimulation frequency electrical stimulation was able to increase strength (9). Carraro et al. (10) suggest that frail persons post-COVID-19 infection with muscle weakness or persons in prolonged inactivity for pandemic-related restriction may benefit from the full-body exercise program associated with NMES. However, these effects are unknown in patients in the acute phase of the disease with severe COVID-19.

The present study aims to describe our clinical protocol in the treatment with neuromuscular electrical stimulation of patients with COVID-19 associated with sepsis and septic shock during their acute intensive care unit stay and to discuss intervention responses in skeletal muscle mass and functional performance.

\section{METHODS}

All participants signed the Informed Consent Term, previously approved by the Ethics Committee of the Hospital Sírio-Libanês (number 3,999,139). This case series was conducted at the adult intensive care units of Hospital Sírio-Libanês, São Paulo, Brazil, and all approved ethical protocols were followed.

\section{Patients}

Seven patients with COVID-19 associated with sepsis or septic shock with age $\geq 18$ years were selected. Sepsis diagnosis was defined by the Third International Consensus Definitions for Sepsis and Septic Shock (Sepsis-3) (11). Furthermore, patients should have the capacity to walk independently before hospitalization classified by mean of the Expanded Disability Status Score $($ EDSS $) \leq 6$ (12) and immobilization period without walking $\leq 7$ days.

\section{Candidate Patients for Neuromuscular Electrical Stimulation}

The inclusion criteria for starting NMES to critically ill patients include body mass index $(\mathrm{BMI}) \leq 35 \mathrm{~kg} / \mathrm{m}^{2}$; without skin lesions, cardiac pacemaker, infection or trauma in lower limbs, neuromuscular diseases, use of neuromuscular blockers, polyneuropathy, and imminent risk of death in less than 48 hours (Simplified Acute Physiology Score III - SAPS III $\leq 80$ ). The exclusion criteria for intervention were infarction and/or need for mechanical cardiopulmonary bypass devices or the need for intra-aortic balloon during ICU hospitalization.

\section{Clinical Assessment}

In the ICU admission, patients were evaluated and classified to clinical severity according to the Simplified Acute Physiology Score III (SAPS III) (13) and the Sequential Organ Failure Assessment (SOFA) (14). In addition, we collected clinical and neurological parameters. SAPS III and SOFA assessments were performed by the medical team of the intensive care unit.

\section{Outcome Measures}

Muscle mass was assessed using ultrasonography. Patients were evaluated concerning rectus femoris cross-sectional area $\left(\mathrm{cm}^{2}\right)$, the thickness of the anterior compartment of the quadriceps muscle (rectus femoris and vastus intermedius) $(\mathrm{cm})$, and rectus femoris echogenicity (pixels) (5). The transducer was positioned perpendicular to the longitudinal axis of the thigh in $80 \%$ of the distal distance between the anterosuperior iliac spine and the upper midpoint of the patella to obtain measurements of the rectus femoris cross-sectional area, and thickness of the anterior compartment of the quadriceps muscle (rectus femoris and vastus intermedius). The measurements were performed using B-mode ultrasound (Logiq e ultrasound, GE Healthcare, USA) (Figure 1).

Functionality was assessed by the International Classification of Functioning, Disability, and Health (ICF) using the muscle strength domains (b730) based on the Medical Research Council (MRC) score for global strength (5). The ICF scores used were: $0-58$ to 60 (without significant changes); $1-48$ to 57 (slight loss); 2-31 to 47 (moderate loss); 3-4 to 30 (severe loss); and 4-0 to 3 (maximum loss). The MRC score is a voluntary method and depends on the understanding and collaboration of the patients. For this reason, in the case of patients on mechanical ventilation, it was evaluated only after interrupting sedation (15). In addition, we assessed functionality by the Physical Function ICU Test-scored (PFIT-s), Morton Mobility Index (DEMMI), and the Surgical Intensive Care Unit Optimal Mobilization Score (SOMS).

PFIT-s examine the capacity of the patient in the sit-tostand level of assistance; maximal marching on the spot duration and number of steps; and shoulder flexion strength, and knee extension strength. The PFIT-s score ranges from 0 (unable to perform activities) to 10 (high physical functioning) (16). DEMMI is composed of 15 hierarchical mobility activities (bedbased, chair-based, static balance, walking-related, and dynamic balance). The total score is converted with Rasch Analysis with a score range from 0 (poor mobility) to 100 (high levels of independent mobility) (16). PFIT-s and DEMMI depend on the understanding and collaboration of the patients, and it was performed after interrupting the sedation. The SOMS score ranges from 0 (indicating that no mobilization should be considered since deemed to be futile, as for patients in a terminal unstable clinical condition such as those with intracranial 

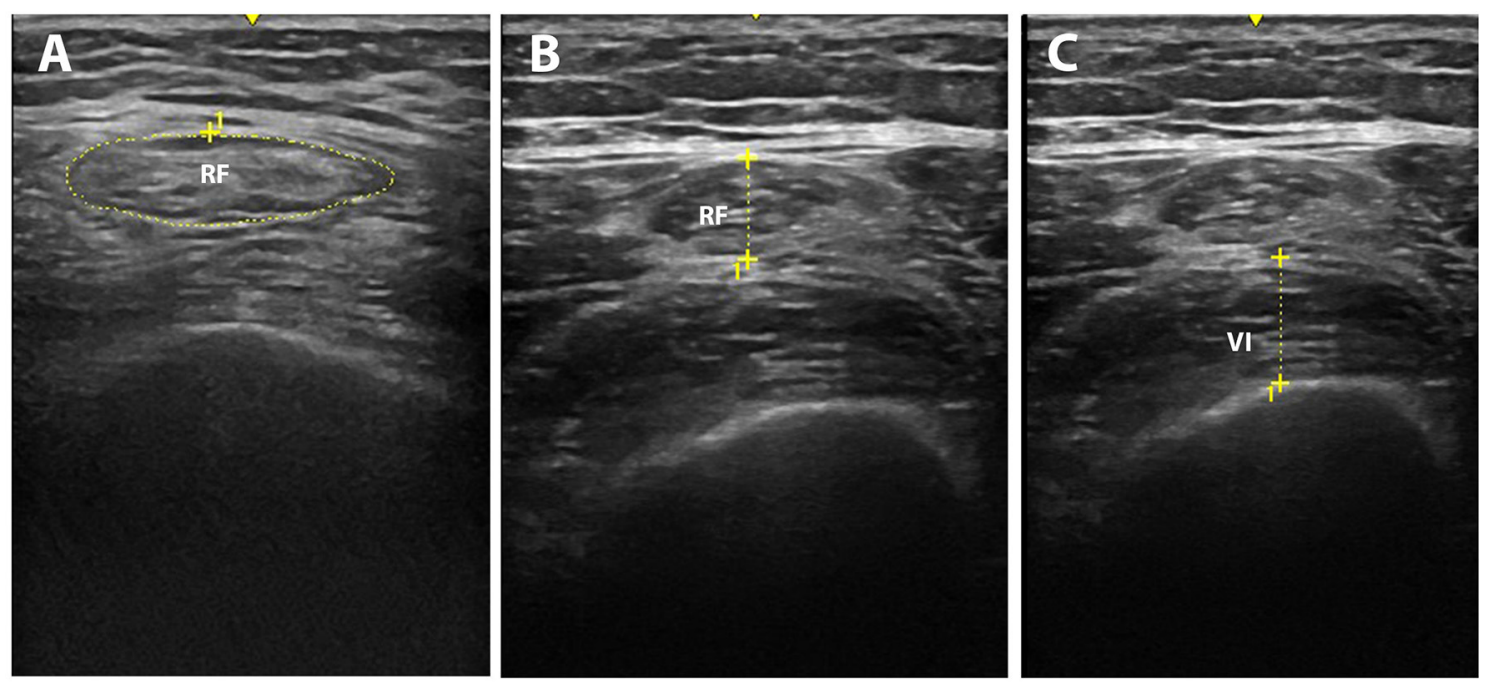

FIGURE 1 | Representative muscle ultrasound image methods: rectus femoris cross-section area (A), the thickness of the anterior compartment of the quadriceps muscle [rectus femoris $\mathbf{( B )}$ and vastus intermedius $\mathbf{( C )}$ ].

hypertension or severe systemic hemodynamic and respiratory insufficiency) to 4 (patients able to ambulate) (17).

The rectus femoris cross-sectional area $\left(\mathrm{cm}^{2}\right)$, the thickness of the anterior compartment of the quadriceps muscle, ICFmuscle strength, PFIT-s, DEMMI, and SOMS were evaluated on days 1, 5, and 8 of start intervention with neuromuscular electrical stimulation. All measurements were performed by the same physiotherapist on days 1,5 , and 8 and was blind to the interventions that were applied to the patients.

\section{Neuromuscular Electrical Stimulation}

The NMES was performed after interrupting the neuromuscular blocker with the patient in the supine position in the ICU bed with 30-60 degrees of the hips and knees joint flexion. The ICU bed itself was used to achieve the positioning of the patient necessary for intervention with NMES. Two pairs of selfadhesive electrodes (size $9 \times 5 \mathrm{~cm}$, SPES Medica Brazil Ltda, São Paulo, Brazil) were positioned distally over the motor area of vastus medialis and vastus lateralis muscles, and the other two were placed $5 \mathrm{~cm}$ below the inguinal region. The location of the electrodes was marked on the skin with a surgical marking pen to ensure application in the same location on subsequent days. This position of the NMES electrodes is capable of stimulating the motor points of the quadriceps muscles (18).

The parameters used were stimulation frequency of $100 \mathrm{~Hz}$, a stimulation pulse width of $350 \mu \mathrm{s}$, a ramp-up time of $1 \mathrm{~s}$, time on of $4 \mathrm{~s}$, ramp-dow of the stimulation of the $1 \mathrm{~s}$, and time off of $12 \mathrm{~s}$. The stimulation pulse width was performed with charge-balanced biphasic pulses and trapezoidal waves. In awake patients, the intensity was established with the maximum muscle contraction tolerated by the patient. In sedated patients, it NMES was adjusted with $50 \%$ above the minimum necessary to generate a visible contraction (8). The stimulation frequency was based on Rodriguez et al. (9) that showed that the high stimulation frequency electrical stimulation presented a preventive effect in the progression of muscle weakness in patients having severe sepsis requiring mechanical ventilation. During the intervention with NMES, no voluntary muscle movement was requested.

The treatment with NMES was interrupted if the patient presented cardiorespiratory instability, high fever (above $39^{\circ} \mathrm{C}$ ), development of muscle fatigue, pain above 7 on the Visual Analog Scale (VAS), or pain above 2 on the Pain Assessment in Advanced Dementia (PAINAD) scale (19).

The application of NMES was carried out by the same physiotherapist on 7 consecutive days in a daily session of 40 minutes. For the treatment, we used the NMES device (Neurodyn II; IBRAMED; Amparo; São Paulo; Brazil). The physiotherapist involved in the NMES intervention did not participate in the outcome assessment and was blind to the results.

\section{Feasibility and Safety}

Feasibility was determined based on adherence and safety was evaluated based on the incidence of adverse events. Adverse events were considered: hemodynamic instability, respiratory instability, skin injury, and bruises.

\section{Statistical Analysis}

Data were assessed for normality using the Shapiro-Wilk test. Parametric variables are presented as mean and standard error. Categorical data are presented as the absolute $(n)$ and relative frequency (\%). Change in the muscle mass and functional capacity was assessed by repeated measure analysis of variance. Statistical significance was indicated by a $P<0.05$.

\section{RESULTS}

Seven patients attended the NMES sessions. One patient stopped the treatment of NMES and one patient died on day 8 (patients 4 and 6); therefore, data for these patients were not included in the outcomes of all patients; only their data are displayed in the 
TABLE 1 | Characteristics of patients with COVID-19 associated with sepsis and septic shock during ICU and hospital stay.

Demographic characteristics and clinical characteristics

\begin{tabular}{|c|c|c|c|c|c|c|c|c|c|c|c|c|c|}
\hline Patient & Age (y) & Gender & BMI & SOFA & SAPS III & $\begin{array}{l}\text { COVID-19 } \\
\text { severity }\end{array}$ & Sedation & $\begin{array}{l}\text { Vasoactive } \\
\text { drug }\end{array}$ & $\begin{array}{l}\text { Neuromuscular } \\
\text { blocker }\end{array}$ & Hydrocortisone & IMV days & ICU stay & $\begin{array}{l}\text { Hospital } \\
\text { stay }\end{array}$ \\
\hline 1 & 67 & Female & 28.9 & 8 & 67 & Critical illness & Yes & Yes & Yes & Yes & 6 & 11 & 24 \\
\hline 2 & 65 & Female & 30.2 & 0 & 38 & Critical illness & Yes & Yes & Yes & Yes & 4 & 15 & 22 \\
\hline 3 & 72 & Male & 30.9 & 7 & 57 & Critical illness & Yes & Yes & Yes & Yes & 8 & 12 & 27 \\
\hline 4 & 61 & Male & 31.7 & 0 & 46 & Critical illness & Yes & Yes & Yes & Yes & 6 & 28 & 28 \\
\hline 5 & 67 & Male & 32.6 & 5 & 50 & Critical illness & No & No & No & Yes & 0 & 9 & 14 \\
\hline 6 & 75 & Male & 31.2 & 7 & 90 & Critical illness & Yes & Yes & Yes & Yes & 9 & 9 & 21 \\
\hline 7 & 70 & Male & 25.8 & 3 & 55 & Critical illness & No & No & No & Yes & 0 & 3 & 11 \\
\hline Mean \pm SD & $68.1 \pm 4.6$ & - & $30.2 \pm 2.3$ & $4.2 \pm 3.3$ & $57.5 \pm 16.9$ & - & - & - & - & - & $4.7 \pm 3.5$ & $12.4 \pm 7.7$ & $21.0 \pm 6.3$ \\
\hline \multicolumn{14}{|c|}{ Neurologic characteristics and comorbidities } \\
\hline Patient & EDSS $\leq 6$ & $\begin{array}{l}\text { RASS } \\
\text { (D1/D5/D8) }\end{array}$ & $\begin{array}{l}\text { Glasgow } \\
\text { (D1/D5/D8) }\end{array}$ & $\begin{array}{l}\text { CAM } \\
\text { (D1/D5/D8) }\end{array}$ & $\begin{array}{l}\text { Oxygen } \\
\text { therapy }\end{array}$ & NIV & Hypertension & $\begin{array}{l}\text { Diabetes } \\
\text { mellitus }\end{array}$ & Obesity & Dyslipidemia & Anxiety & Hypothyroidism & COPD \\
\hline 1 & Yes & $-2 / 0 / 0$ & $\bullet / 15 / 15$ & $-/-/-$ & Yes & Yes & No & No & Yes & No & No & Yes & No \\
\hline 2 & Yes & $1 / 0 / 0$ & $\bullet / 15 / 15$ & $-/-/-$ & Yes & Yes & Yes & No & Yes & No & No & No & No \\
\hline 3 & Yes & $1 / 0 / 0$ & $\bullet / 15 / 15$ & $+/-/-$ & Yes & No & No & No & No & Yes & No & Yes & No \\
\hline 4 & Yes & $-4 / 0 / \bullet$ & $\bullet / 15 / \bullet$ & $-/-/ \bullet$ & Yes & No & No & No & No & No & No & No & No \\
\hline 5 & Yes & 0/0/0 & 15/15/15 & $-/-1-$ & Yes & No & Yes & Yes & Yes & Yes & No & No & No \\
\hline 6 & Yes & $-5 /-5 / \bullet$ & $\bullet / \bullet$ & $-/-/ \bullet$ & Yes & Yes & Yes & No & Yes & Yes & No & No & No \\
\hline 7 & Yes & 0/0/0 & $15 / 15 / 15$ & $-/-/-$ & Yes & Yes & Yes & No & No & No & No & No & Yes \\
\hline
\end{tabular}

BMI, body mass index; CAM, Confusion Assessment Method; COPD, chronic obstructive pulmonary disease; EDSS, Expanded Disability Status Score; ICU, intensive care unit; IMV, invasive mechanical ventilation; NIV, non-invasive ventilation; RASS, Richmond Agitation Sedation Scale; SAPS III, Simplified Acute Physiology Score III; SOFA, Sequential Organ Failure Assessment; • not applicable. 

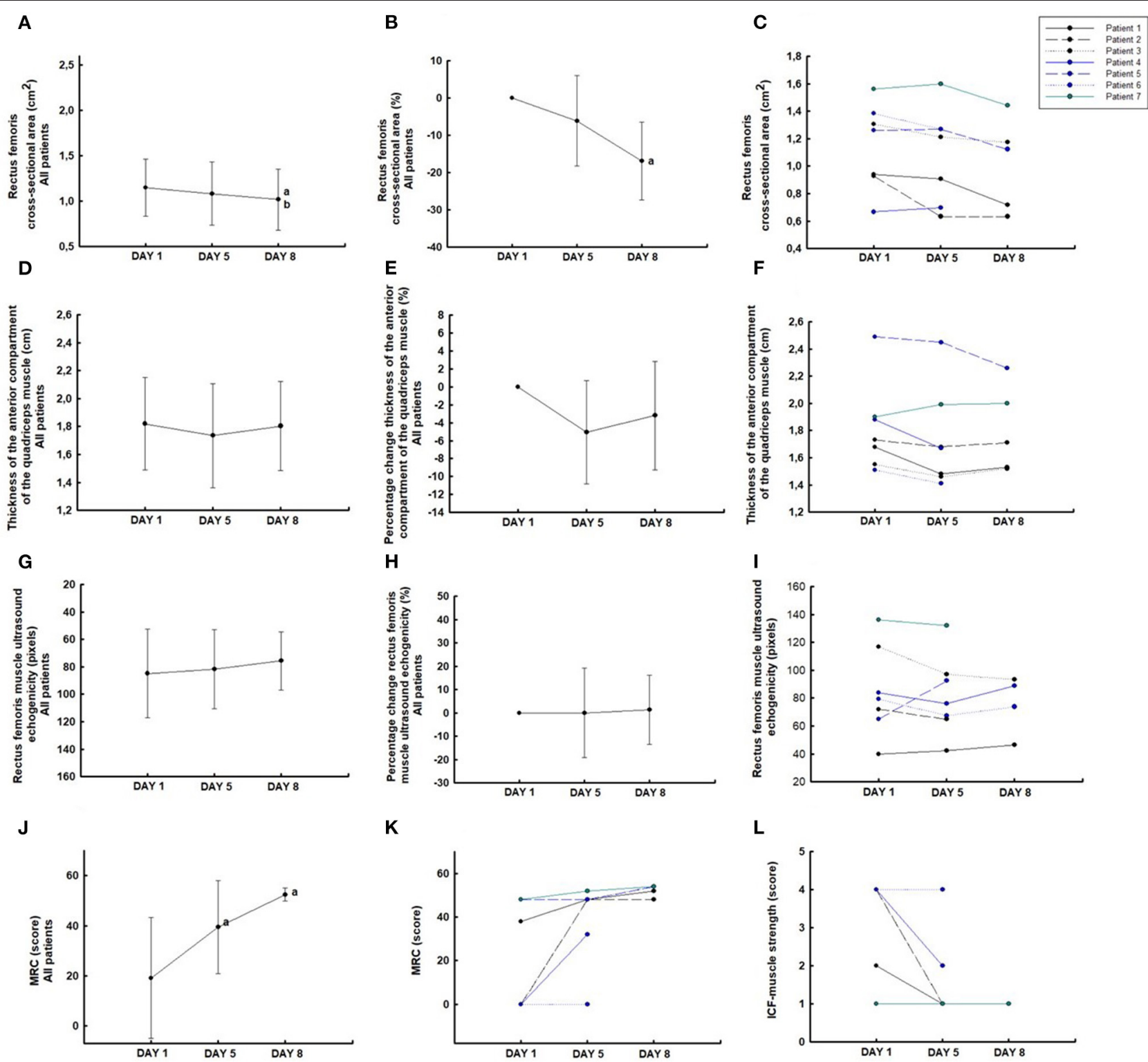

K

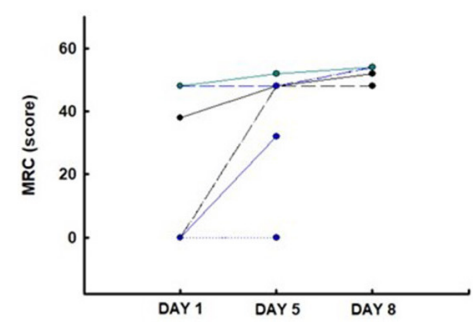

L

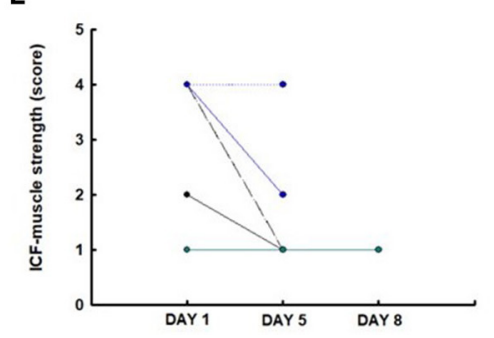

FIGURE 2 | Ultrasound muscle assessement of the rectus femoris cross-section área (A-C); the thickness of the anterior compartment of the quadriceps muscle (rectus femoris and vastus intermedius) (D-F); rectus femoris echogenicity (G-I); MRC score (J,K); and International Classification of Functioning, Disability, and Health (ICF)-muscle strength (L). a $P<0.05$ compared to day 1 ; ${ }^{b} P<0.05$ compared to day 5.

individual patient values graph. The demographic characteristics of the patients are shown in Table $\mathbf{1}$.

\section{Muscle Mass Outcomes}

The rectus femoris cross-sectional area decreased significantly $(-16.9 \%$ [95\% CI, -29.8 to -3.9 ]; $P<0.05)$ from days 1 to 8 (Figures 2A-C), but showed maintenance of the thickness of the anterior compartment of the quadriceps muscle $(-3.20 \%$ [95\% $\mathrm{CI},-10.6$ to 4.2 ]; $P=0.3$ ) from days 1 to 8 (Figures $2 \mathrm{D}-\mathbf{F}$ ). These patients showed a reduction of $2.1 \%$ [ $95 \% \mathrm{CI},-3.7$ to -0.5$]$ per day in the rectus femoris cross-sectional area and 0.3\% [95\% CI, -1.3 to 0.5 ] per day in the thickness of the anterior compartment of the quadriceps muscle during 8 days. Furthermore, patients showed maintenance of the echogenicity $(1.3 \%$ [95\% CI, -17.1 to $19.7 \%$ ]; $P=0.8$ ) from days 1 to 8 with an increase of $0.16 \%$ per day (Figures 2G-I).

\section{Peripheral Muscle Strength and Functional Outcomes}

The MRC score increased significantly from days 1 to $5(P<0.05)$ and kept this improvement until day $8(P=0.5)$ (Figure 2J). In the five patients evaluated, all (100\%) showed an increase in the MRC score (Figure $2 \mathbf{K}$ ) and reduction of the ICF-muscle strength, meaning improved muscle strength from days 1 to 8 

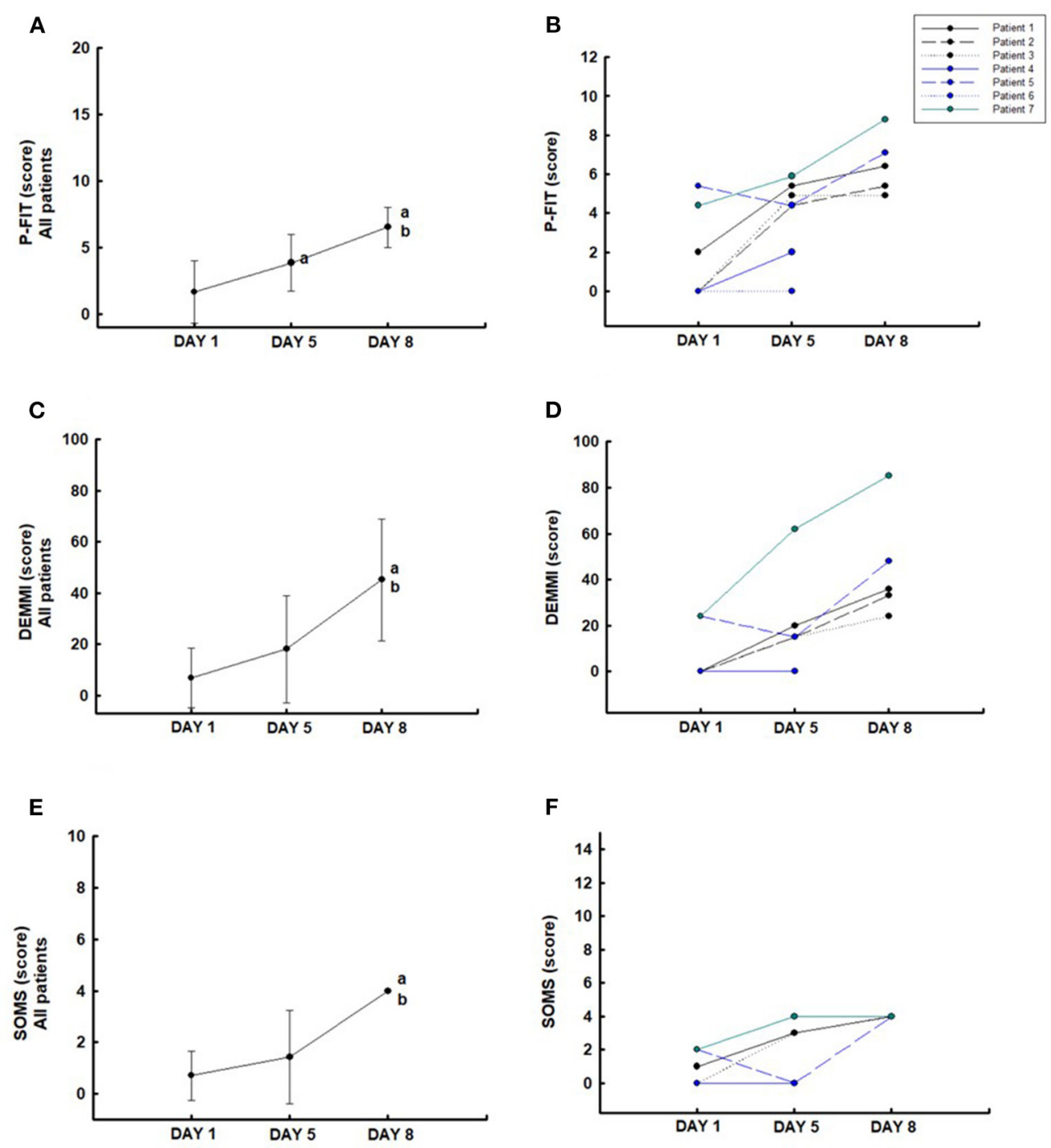

FIGURE 3 | Functionality: Physical Function ICU Test-scored (PFIT-s) (A,B), Morton Mobility Index (DEMMI) (C,D), and the Surgical Intensive Care Unit Optimal Mobilization Score (SOMS) (E,F). ${ }^{\mathrm{a}} P<0.05$ compared to day 1 ; ${ }^{\mathrm{b}} P<0.05$ compared to day 5 .

(Figure 2L). Four (80\%) patients evaluated showed an increase in the MRC score and one (20\%) maintained the MRC score values from days 5 to 8 . Three patients $(60 \%)$ showed a decrease in the ICF-muscle strength from days 1 to 5 and these values were maintained on day 8 . Two patients (40\%) maintained the ICF-muscle strength on days 5 and 8 compared with the baseline values (day 1 ).

The PFIT-s increased significantly from days 1 to 5 and improved until day 8 compared to day $5(P<0.05)$ (Figure 3A). All patients $(100 \%)$ showed an increase in the PFIT-s on day 5 compared to day 1 and improvement on day 8 compared to day 5 (Figure 3B). DEMMI (Figure 3C) and SOMS (Figure 3E) scores increased significantly on day 8 compared to days 1 and $5(P<0.05)$. In the five patients evaluated, the individual data present that all (100\%) patients showed an increase in the DEMMI (Figure 3D) and SOMS (Figure 3F) scores on days 5 and 8 compared with the baseline values (day 1).

\section{Feasibility and Safety}

No adverse events were reported during the case series. Five patients completed the assessments and intervention. One patient interrupted the NMES intervention but did not claim intolerance during the application, and one patient died due to worsening pulmonary and respiratory conditions. None of the NMES intervention sessions were interrupted by pain.

\section{DISCUSSION}

Patients with COVID-19 associated with sepsis and septic shock treated with NMES presented a reduction of $16.9 \%$ in the rectus 
femoris cross-sectional area, but with no significant reduction in the thickness of the anterior compartment of the quadriceps muscle (3.2\%) and no significant increase of rectus femoris echogenicity on day $8(1.3 \%)$. The magnitude of these alterations was $2.1,0.3$, and $0.16 \%$ per day, respectively. We emphasize that these reported values in the present case series are smaller than those found compared to another study conducted at the same hospital and research group that evaluated severe COVID19 patients without NMES intervention. This study showed a reduction of $30.1 \%$ in the rectus femoris cross-sectional area, $18.6 \%$ in the thickness of the anterior compartment of the quadriceps muscle, and increase of $16.8 \%$ in the echogenicity on day 10 with themagnitude of these alterations being about 3.7, 2.1 , and $1.68 \%$ per day, respectively (5).

The ability of electrical muscle stimulation to improve or maintain strength, muscle mass, and functionality in ICU patients with sepsis is controversial. However, the results seem to be related to the type of stimulation frequency involved in muscle stimulation. Rodriguez et al. (9) used high stimulation frequency in the neuromuscular electrical stimulation and showed a preventive effect in the progression of muscle weakness in patients having severe sepsis requiring mechanical ventilation. On the other hand, when Poulsen et al. (8) used low stimulation frequency in the patients with septic shock admitted to the ICU, and showed that loss of muscle mass was unaffected by electrical muscle stimulation. Our results corroborate with the Rodriguez et al. (9) study and enhance the possible benefit of using high stimulation frequency for muscle electrical stimulation.

The effect of electrical muscle stimulation on muscle mass and strength can be explained by several factors. Nuhr et al. (20) and Hambrecht et al. (21) showed that NMES induces an increase in oxidative capacity with the transition from fast to slow fiber types associated with a decrease in anaerobic enzymes levels. All physiological muscle changes found with the use of electrical muscle stimulation in critically ill patients suggest that the origin is a systemic effect on microcirculation (22). Vanderthommen et al. (23) showed that in the identical levels of workload ( $10 \%$ of the quadriceps maximum isometric voluntary torque), the muscle reaches higher values in blood flow and oxygen consumption during NMES compared with voluntary muscle contractions. Moreover, a single session of NMES is sufficient to stimulate the increased levels of mRNA for IGF binding protein-4 (84\%), MyoD (83\%), myogenin ( 3fold), cyclin D1 (50\%), and p21-Waf1 (16-fold), which are indicative of the initiation of myogenic processes in skeletal muscle. In the same study, an additional NMES session (a total of 14 min spread over 2 days), was sufficient to induce an increase in the concentration of total skeletal muscle ribonucleic acid (RNA) (24), most likely representing an increase in muscle protein synthesis. These results indicate that molecular adaptations of skeletal muscle to loading respond in a very short time.

Neuromuscular blocking agents cause skeletal muscle relaxation by blocking the transmission of impulses at the neuromuscular junction (25). NMES evokes a muscle contraction by activating intramuscular branches of the nerve to the muscle and not the muscle fibers directly (26) and selected brain regions in a doseresponse manner (27). The use of neuromuscular blocking agents during NMES intervention may interfere with the performance of muscle contraction. However, neuromuscular blockers present a recovery time of 8$40 \mathrm{~min}$ after their interruption (28). Therefore, we performed NMES intervention after interrupting the neuromuscular blocking agents.

Sedation is commonly used in patients admitted to the intensive care unit (29). Dirkes et al. (30) showed that NMES represents an effective and feasible interventional strategy to prevent skeletal muscle atrophy in a fully sedated patient with critically ill. In the same study, the non-stimulated leg showed substantial type 1 and type 2 muscle fiber atrophy (a $16 \pm 9$ and $24 \pm 7 \%$ decline in muscle fiber; respectively). In contrast, no atrophy was observed in the muscle fibers collected from the stimulated leg. Although sedation does not interfere with NMES intervention, it can compromise functional assessments. Therefore, in the present study, the MRC score, P-FITs, and DEMMI evaluations were performed only after sedation withdrawal.

The limitation of this study is that it is a single-center study design and there is no control group to compare the efficacy. In addition, the number of cases is small and it is unclear whether the results can be generalized. Mateo et al. (31) used functional electrical stimulation associated with cycling in patients post-hospitalization in the ICU for a critical form of COVID-19. However, the present case series is the first report of the effects of neuromuscular electrical stimulation intervention in patients with severe COVID-19 in the acute phase of the disease associated with sepsis and septic shock. Randomized clinical trials with more patients reporting the efficacy of electrical stimulation using NMES in patients with COVID-19 associated with sepsis and septic shock are needed to confirm our findings.

\section{CONCLUSION}

Rehabilitation with NMES showed improvement in muscle strength and functionality of patients in this case series with a potential protective effect on muscle mass loss in patients with critical COVID-19 associated with sepsis and septic shock.

\section{DATA AVAILABILITY STATEMENT}

The raw data supporting the conclusions of this article will be made available by the authors, without undue reservation.

\section{ETHICS STATEMENT}

The studies involving human participants were reviewed and approved by Ethics Committee of the Hospital SírioLibanês (number 3,999,139). The patients/participants 
provided their written informed consent to participate in this study.

\section{AUTHOR CONTRIBUTIONS}

SG, WC, LS, IS, and WY: study design. SG, WC, and LS: data collection. RR and WY: data analysis and draft manuscript. RR, SG, WC, LS, IS, and WY: manuscript review.

\section{REFERENCES}

1. World Health Organization. Clinical Management of COVID-19. May 14, 2020. (2020). Available online at: https://www.who.int/publications/i/item/ clinical-management-of-covid-19 (accessed October 1, 2020)

2. Lazzeri M, Lanza A, Bellini R, Bellofiore A, Cecchetto S, Colombo A, et al. Respiratory physiotherapy in patients with COVID-19 infection in acute setting: a Position Paper of the Italian Association of Respiratory Physiotherapists (ARIR). Monaldi Arch Chest Dis. (2020) 90:163-168. doi: 10.4081/monaldi.2020.1285

3. Righetti RF, Onoue MA, Politi FVA, Teixeira DT, Souza PN, Kondo CS, et al. Physiotherapy care of patients with coronavirus disease 2019. (COVID-19) - a Brazilian experience. Clinics. (2020) 75:e2017. doi: 10.6061/clinics/2020/e2017

4. Guan WJ, Ni ZY, Hu Y, Liang WH, Ou CQ, He JX, et al. Clinical characteristics of Coronavirus Disease 2019 in China. New Engl J Med. (2020) 382:170820. doi: 10.1056/NEJMoa2002032

5. de Andrade-Junior MC, de Salles ICD, de Brito CMM, Pastore-Junior L, Righetti RF, Yamaguti WP. Skeletal muscle wasting and function impairment in intensive care patients with severe COVID-19. Front Physiol. (2021) 12:640973. doi: 10.3389/fphys.2021.640973

6. Vanhorebeek I, Latronico N, Van den Berghe G. ICU-acquired weakness. Intensive Care Med. (2020) 46:637-53. doi: 10.1007/s00134-020-05944-4

7. Nakanishi N, Oto J, Tsutsumi R, Yamamoto T, Ueno Y, Nakataki E, et al. Effect of electrical muscle stimulation on upper and lower limb muscles in critically ill patients: a two-center randomized controlled trial. Crit Care Med. (2020) 48:e997-e1003. doi: 10.1097/CCM.0000000000004522

8. Poulsen JB, Møller K, Jensen CV, Weisdorf S, Kehlet H, Perner A. Effect of transcutaneous electrical muscle stimulation on muscle volume in patients with septic shock. Crit Care Med. (2011) 39:456-61. doi: 10.1097/CCM.0b013e318205c7bc

9. Rodriguez PO, Setten M, Maskin LP, Bonelli I, Vidomlansky SR, Attie S, et al. Muscle weakness in septic patients requiring mechanical ventilation: protective effect of transcutaneous neuromuscular electrical stimulation. J Crit Care. (2012) 27:319.e1-8. doi: 10.1016/j.jcrc.2011.04.010

10. Carraro U, Marcante A, Ravara B, Albertin G, Maccarone MC, Piccione F, et al. Skeletal muscle weakness in older adults home-restricted due to COVID-19 pandemic: a role for full-body in-bed gym and functional electrical stimulation. Aging Clin Exp Res. (2021) 33:20539. doi: 10.1007/s40520-021-01885-0

11. Singer M, Deutschman CS, Seymour CW, Shankar-Hari M, Annane D, Bauer $\mathrm{M}$, et al. The third international consensus definitions for sepsis and septic shock (Sepsis-3). JAMA. (2016) 315:801-10. doi: 10.1001/jama.2016.0287

12. Kurtzke JF. Rating neurologic impairment in multiple sclerosis: an expanded disability status scale (EDSS). Neurology. (1983) 33:144452. doi: 10.1212/WNL.33.11.1444

13. Moreno RP, Metnitz PG, Almeida E, Jordan B, Bauer P, Campos RA, et al. SAPS 3-From evaluation of the patient to evaluation of the intensive care unit. Part 2: development of a prognostic model for hospital mortality at ICU admission. Intensive Care Med. (2005) 31:134555. doi: 10.1007/s00134-005-2763-5

14. Vincent JL, Moreno R, Takala J, Willatts S, De Mendonça A, Bruining H, et al. The SOFA (Sepsis-related Organ Failure Assessment) score to describe organ dysfunction/failure. on behalf of the working group on sepsis-related problems of the European society of intensive care medicine. Intensive Care Med. (1996) 22:707-10. doi: 10.1007/BF01709751
All authors contributed to the article and approved the submitted version.

\section{ACKNOWLEDGMENTS}

The authors thank the Fundação de Amparo à Pesquisa do Estado de São Paulo FAPESP, number 18/16832-9) and Hospital Sírio-Libanês.

15. Hermans G, Clerckx B, Vanhullebusch T, Segers J, Vanpee G, Robbeets C, et al. Interobserver agreement of Medical Research Council sum-score and handgrip strength in the intensive care unit. Muscle Nerve. (2012) 45:1825. doi: $10.1002 /$ mus. 22219

16. Silva VZMD, Lima AS, Nadiele $H$, Pires-Neto $R$, Denehy L, Parry SM. Brazilian versions of the physical function icu testscored and de morton mobility index: translation, cross-cultural adaptation, and clinimetric properties. J Bras Pneumol. (2020) 46:e20180366. doi: 10.36416/1806-3756/e20180366

17. Kasotakis G, Schmidt U, Perry D, Grosse-Sundrup M, Benjamin J, Ryan C, et al. The surgical intensive care unit optimal mobility score predicts mortality and length of stay. Crit Care Med. (2012) 40:11228. doi: 10.1097/CCM.0b013e3182376e6d

18. Botter A, Oprandi G, Lanfranco F, Allasia S, Maffiuletti NA, Minetto MA. Atlas of the muscle motor points for the lower limb: implications for electrical stimulation procedures and electrode positioning. Eur J Appl Physiol. (2011) 111:2461-71. doi: 10.1007/s00421-011-2093-y

19. Valera GG, Carezzato NL, Vale FA, Hortense P. Adaptação cultural para o Brasil da escala Pain Assessment in Advanced Dementia PAINAD* [Cultural adaptation of the scale Pain Assessment in Advanced Dementia - PAINAD to Brazil]. Rev Esc Enferm USP. (2014) 48:4628. doi: 10.1590/S0080-623420140000300011

20. Nuhr M, Crevenna R, Gohlsch B, Bittner C, Pleiner J, Wiesinger $\mathrm{G}$, et al. Functional and biochemical properties of chronically stimulated human skeletal muscle. Eur J Appl Physiol. (2003) 89:202-8. doi: 10.1007/s00421-003-0792-8

21. Hambrecht R, Fiehn E, Yu J, Niebauer J, Weigl C, Hilbrich L, et al. Effects of endurance training on mitochondrial ultrastructure and fiber type distribution in skeletal muscle of patients with stable chronic heart failure. $J$ Am Coll Cardiol. (1997) 29:1067-73. doi: 10.1016/S0735-1097(97)00015-6

22. Gerovasili V, Tripodaki E, Karatzanos E, Pitsolis T, Markaki V, Zervakis D, et al. Short-term systemic effect of electrical muscle stimulation in critically ill patients. Chest. (2009) 136:1249-56. doi: 10.1378/chest.08-2888

23. Vanderthommen M, Duteil S, Wary C, Raynaud JS, Leroy-Willig A, Crielaard $\mathrm{JM}$, et al. A comparison of voluntary and electrically induced contractions by interleaved 1H- and 31P-NMRS in humans. J Appl Physiol. (2003) 94:101224. doi: 10.1152/japplphysiol.00887.2001

24. Bickel CS, Slade J, Mahoney E, Haddad F, Dudley GA, Adams GR. Time course of molecular responses of human skeletal muscle to acute bouts of resistance exercise. J Appl Physiol. (2005) 98:482-8. doi: 10.1152/japplphysiol.00895.2004

25. Renew JR, Ratzlaff R, Hernandez-Torres V, Brull SJ, Prielipp RC. Neuromuscular blockade management in the critically Ill patient. J Intensive Care. (2020) 8:37. doi: 10.1186/s40560-020-00455-2

26. Collins DF. Central contributions to contractions evoked by tetanic neuromuscular electrical stimulation. Exerc Sport Sci Rev. (2007) 35:1029. doi: 10.1097/jes.0b013e3180a0321b

27. Smith GV, Alon G, Roys SR, Gullapalli RP. Functional MRI determination of a dose-response relationship to lower extremity neuromuscular electrical stimulation in healthy subjects. Exp Brain Res. (2003) 150:33-9. doi: 10.1007/s00221-003-1405-9

28. Moore EW, Hunter JM. The new neuromuscular blocking agents: do they offer any advantages? Br J Anaesth. (2001) 87:912-25. doi: 10.1093/bja/87.6.912

29. Marra A, Ely EW, Pandharipande PP, Patel MB. The ABCDEF bundle in critical care. Crit Care Clin. (2017) 33:225-43. doi: 10.1016/j.ccc.2016.12.005 
30. Dirks ML, Hansen D, Van Assche A, Dendale P, Van Loon LJ. Neuromuscular electrical stimulation prevents muscle wasting in critically ill comatose patients. Clin Sci (Lond). (2015) 128:357-65. doi: 10.1042/CS201 40447

31. Mateo S, Bergeron V, Cheminon M, Guinet-Lacoste A, Pouget MC, Jacquin-Courtois S, et al. Functional electrical stimulation-cycling favours erectus position restoration and walking in patients with critical COVID19. a proof-of-concept controlled study. Ann Phys Rehabil Med. (2021) 64:101516. doi: 10.1016/j.rehab.2021.101516

Conflict of Interest: The authors declare that the research was conducted in the absence of any commercial or financial relationships that could be construed as a potential conflict of interest.
Publisher's Note: All claims expressed in this article are solely those of the authors and do not necessarily represent those of their affiliated organizations, or those of the publisher, the editors and the reviewers. Any product that may be evaluated in this article, or claim that may be made by its manufacturer, is not guaranteed or endorsed by the publisher.

Copyright (c) 2022 Righetti, Grams, Costa, Saraiva, Salles and Yamaguti. This is an open-access article distributed under the terms of the Creative Commons Attribution License (CC BY). The use, distribution or reproduction in other forums is permitted, provided the original author(s) and the copyright owner(s) are credited and that the original publication in this journal is cited, in accordance with accepted academic practice. No use, distribution or reproduction is permitted which does not comply with these terms. 